\title{
Reduced oscillations based perturb and observe solar maximum power point tracking scheme to enhance efficacy and speed of a photovoltaic system
}

\section{DOI : 10.36909/jer.13569}

\author{
Pawan Kumar Pathak*, Anil Kumar Yadav** and P. A. Alvi**** \\ ${ }^{*}$ School of Automation, Banasthali Vidyapith, Rajasthan, 304022, India. \\ ${ }^{* * *}$ Department of Electrical Engineering, National Institute of Technology Hamirpur, Hamirpur (H.P.) 177005, India. \\ *** Department of Physics, Banasthali Vidyapith, Rajasthan, 304022, India.
}

Corresponding Author: ppathak999@gmail.com

\begin{abstract}
This paper puts forward a reduced oscillation based perturb and observe (ROP\&O) maximum power point (MPP) tracking (MPPT) technique to mitigate the probability of loss of tracking direction and to reduce oscillations around MPP when the solar photovoltaic (PV) array is subjected under periodically changing irradiance. The proposed technique retains the structure of conventional perturb and observe $(\mathrm{P} \& \mathrm{O})$ technique additionally incorporating a unique structure of dynamic step sizing, along with proportional-integral (PI) controller which potently alters the duty cycle $(D)$ of the DC-DC boost power converter (BPC). The ROP\&O MPPT technique is compared with conventional P\&O and incremental conductance (IC) schemes in terms of tracking efficacy $(\eta)$, ripples in PV voltage and PV current, convergence time, and the error rates. The efficacy of the proposed scheme lies between $99.06 \%$ to $99.80 \%$. Moreover, the time to obtain MPP is 0.018 sec. which is about five times faster than the $\mathrm{P} \& \mathrm{O}$ technique and fifteen times faster than the IC technique. Also, the proposed MPPT technique is benchmarked using three-phase grid integration, and the power quality of the grid current is observed in terms of total harmonic distortion (THD).
\end{abstract}

Keywords: Reduced oscillations based perturb and observe (ROP\&O) MPPT technique; Solar photovoltaic (PV) system; Boost converter; Grid Integration.

\section{Introduction}

For the sustainable supply of energy, low carbon emission, and reduction in dependency on fossil fuels, it is indispensable to exploit and promote renewable sources on a large scale (Ramesh et al., 2021(a)). Among all the renewable sources, PV is one of the most potent forms due to its availability, installation ease, free from moving parts, and simple maintenance (Pathak \& Yadav, 2019). Due to the weather condition's dependency, the PV system suffers from low efficacy, making it costlier than fossil fuels (Soon \& Mekhilef, 2015). One efficient way to enhance $\eta$ is to utilize a significant MPPT technique, which would fruitfully provide maximum obtainable power from the PV. The approach of the MPPT technique to enhance energy throughput is very economical because it comprises software codes. The function of MPPT is to assure that the PV voltage $\left(\mathrm{V}_{\mathrm{pv}}\right)$ and PV current $\left(\mathrm{I}_{\mathrm{pv}}\right)$ always stay at MPP on the P-V characteristic curve (Reisi et al., 2013). To date, innumerable MPPT schemes are reported (Reisi et al., 2013; Pathak et al., 2020; Pathak et al., 2021(a)), which are mainly categorized into two broad categories, conventional and soft computing-based strategies. The most admired conventional MPPT techniques are P\&O (Ahmed \& Salam 2016; Ali et al., 2018; Alik \& Jusoh 2017; Femia et al., 2005; Kumar et al., 2018), and IC (Danandeh et al., 2018; Elgendy et al., 2013; Safari \& Mekhilef, 2010; Pathak et al., 2021(b); Ramesh et al., 2021(b)). These techniques are extensively implemented in commercial products due to their robustness 
and ease of implementation. Moreover, soft computing based MPPT techniques (Pathak et al., 2021(a)) is more accomplished and flexible, despite showing good steady-state (SS) performance, they are very sluggish, complicated, and unacceptable. Due to the complexity level and computational load, the microcontroller required for the soft computing based MPPTs is sophisticated as compared to the conventional MPPTs.

Among all the conventional MPPT techniques, the most popular, most straightforward and cost-effective MPPT technique is $\mathrm{P} \& \mathrm{O}$, and it also exhibits good convergence. The microcontroller required for the P\&O MPPT technique's hardware implementation is unsophisticated and straightforward. Considering these advantages, the proposed ROP\&O MPPT technique is rooted on classical P\&O MPPT technique. However, the classical P\&O MPPT technique suffers from two severe drawbacks of endless oscillations around the MPP and loss of tracking direction during large/steep insolation change (Li et al., 2016). Due to these drawbacks, there is a loss in obtainable power, which subsequently reduces the $\eta$. Even though several works address the problem of oscillation with the adaptive P\&O technique (Kollimalla $\&$ Mishra 2016(a); Zhang et al., 2013), none has elaborately discussed the tracking direction loss issue despite being highlighted (Elgendy etal., 2012). Notwithstanding this, (Kollimalla \& Mishra 2016(b); Paz \& Ordonez 2014; Killi \& Samanta 2015) have discussed several techniques to address these two difficulties. However, the schemes are narrow for particular conditions. The adaptive $\mathrm{P} \& \mathrm{O}$ technique, due to aggressive derivatives, suffers from high computational load (Pandey et al., 2008), and by selecting perturb, still, oscillation around MPP is present during tracking and SS operation (Abdelsalam et al., 2011). With this hindsight, this paper puts forward a more comprehensive ROP\&O MPPT technique aiming to solve both the problems concurrently fruitfully. The ROP\&O scheme retains the structural similarity of the conventional $\mathrm{P} \& \mathrm{O}$ besides incorporating a unique structure of dynamic step sizing and PI controller in the loop to mitigate the oscillations while maintaining excellent convergence time. To prevent overshoot at starting (at the time of tracking) and remove SS oscillations, PI controller is utilized. The PI controller used for the generation of appropriate gating signal for the converter. Its function is to enforce the actual PV voltage $\left(V_{p v}\right)$ to track the reference MPP voltage $\left(V_{r e f, m p p}\right)$ of the PV array. Gain-margin (GM) and phase-margin (PM) criterion are utilized to obtain the PI controller parameters.

A $200 \mathrm{~W}$ prototype of the proposed work is designed, simulated, and benchmarked using the MATLAB/Simulink software. The obtained results of ROP\&O MPPT technique are vividly compared with designed pre-existing conventional P\&O and conventional IC MPPT techniques in terms of $\eta$, convergence time, the ripple in $V_{p v}$ and $I_{p v}$, mean absolute percentage error (MAPE), mean relative error (MRE), and root mean square error (RMSE). The presented methodology features adaptive tracking, quite high $\eta$, reduced oscillations around MPP, less fluctuation in $V_{p v}$ and $I_{p v}$, and adaptability with a large range of operating points of the PV system. At last, the proposed MPPT scheme is validated using three phase grid integration and the power quality of the injected grid current is observed. The prime contributions of the proposed work are summarized as:

- Design and implementation of ROP\&O MPPT scheme in stochastic solar insolation level i.e., $400-1000 \mathrm{~W} / \mathrm{m}^{2}$ at $25^{\circ} \mathrm{C}$.

- The proposed scheme's obtained results are vividly compared with designed conventional P\&O and IC MPPT schemes.

- The stability analysis of the PV fed BPC employing ROP\&O as an MPPT scheme.

\section{Description of PV system}

PV panels are the key elements of the PV power system, which are generally connected in shunt and/or series in order to enhance their power output and generated voltage. The BPC is utilized for impedance matching to harvest maximum obtainable power from the PV panel. The conventional BPC retains the advantages of less component and structural simplicity compared to other special type DC-DC converters (Pathak et al., 2019; Kollimalla \& Mishra 2016(a); Kollimalla \& Mishra 2016(b)), so considered in this work. Controlling the BPC inculcates MPPT and PWM technique, 
which transfers the MPPT control signal to generate the BPC 's gating pulse. A 200W PV panel is selected in this presented work, and simulation of the PV system employing ROP\&O as the MPPT technique is performed using MATLAB/Simulink using three series-connected PV cells. The proposed MPPT scheme is schematically represented in Fig. 1. For the modeling purpose a one diode model is used in this work (Pathak et al., 2019; Ding et al., 2012).

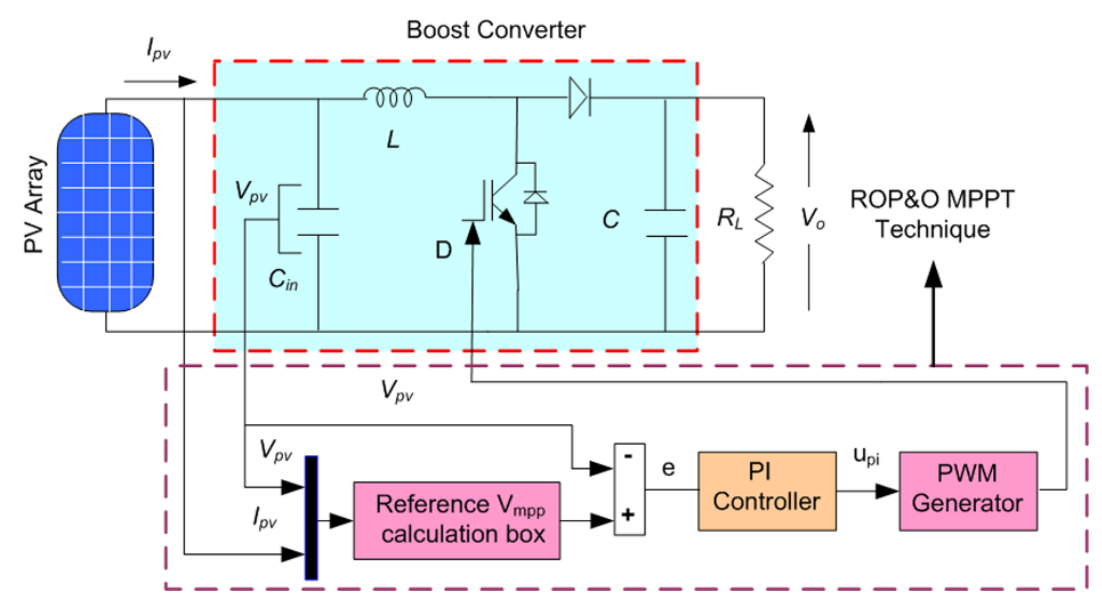

Fig. 1 Schematic presentation of the proposed MPPT technique along

\section{A) Modeling of BPC}

DC voltage source from the PV panel acts as an input for it. Converter output is controlled through $D$ of the IGBT, which is in turn controlled through PWM (Kollimalla \& Mishra 2016(b)). Converter $D$ is a very important element for selecting it's components and harvesting the maximum obtainable power from the PV panel (Pathak and Yadav, 2019). The output voltage $\left(V_{o}\right)$ and input voltage $\left(V_{i n}\right)$ of the BPC is associated as:

$V_{o}=\frac{V_{\text {in }}}{(1-D)}$

Voltage at MPP $\left(V_{m p p}=25.92 \mathrm{~V}\right)$ works as $V_{i n}$, and the BPC is designed for the $V_{o}$ of $36 \mathrm{~V}$, so the obtained $D$ from (1) is 0.28. Current at MPP $\left(I_{m p p}=7.7 A\right)$ works as input current $\left(I_{\text {in }}\right)$ of the BPC, so the output current of the BPC $\left(I_{o}\right)$ is given as (Pathak and Yadav, 2019):

$I_{o}=(1-D) I_{\text {in }}$

From (2) obtained $I_{o}$ is 5.544A. The inductance $(L)$ of the BPC is calculated as (Pathak and Yadav, 2019):

$L=\frac{V_{i n^{*}} \text { D }}{f_{s} * \Delta I}$

where, $\Delta I$ is the ripple content in $I_{o}$ (considered as $5 \%$ of $I_{o}$ ), and $f_{s}(10 \mathrm{kHz})$ is the switching frequency. From (3) obtained value of $L$ is $2.62 \mathrm{mH}$. The capacitance of the BPC $C$ is formulated as (Pathak et al., 2019):

$C=\frac{I_{O} * D}{f_{S} * \Delta V}$

where, $\Delta V$ is the voltage ripple in $V_{o}$ (considered as $2 \%$ of $\left.V_{o}\right)$, the obtained value of $C$ is $215.6 \mu F$. Load resistance $\left(R_{L}=\right.$ $\left.\frac{V_{o}}{I_{o}}=6.5 \Omega\right)$. The $D$ corresponds to the MPP while neglecting the power losses in converter, can be calculated as:

$D_{m p p}=1-\frac{V_{m p p}}{\sqrt{P_{m p p} \times R_{L}}}=0.28$

\section{MPP extraction techniques}

There is an exclusive point on P-V characteristic curve called MPP, whose location shifts based on the environmental conditions. This leads to a major setback of the PV system of having low $\eta$. To enhance the efficacy, continuous tracking of MPP is necessary, which is achieved by implementing a suitable MPPT technique with the help of a boost converter. The unique point of MPP on the characteristic curve is shown in Fig. 2. 


\section{A) P\&O technique}

$V_{p v}$ and $I_{p v}$ are sensed, and power is calculated at every distinct point of the PV array, with the consideration of desired voltage variations. If the power is larger than the previous power corresponding to the new array voltage, then process is continued in the same direction; while, the direction is reversed. This process is continued until maximum point is achieved. At the insolation level of $S$, let point $A$ is the operating point on the $\mathrm{P}-\mathrm{V}$ characteristic curve at the voltage level of $V_{1}$, as shown in Fig. 2. The nature of voltage perturbation $\Delta V_{1}$ (either + or -) is decided based on the sign of $\frac{\Delta P}{\Delta V}$. From point $A$ to point $B$ with positive perturbation $\Delta V_{1}, \frac{\Delta P_{1}}{\Delta V_{1}}$ is positive in nature. Thus, the voltage of the PV module is further perturbed from point $B$ to point $C$ in the same direction. This process is continued till $\frac{\Delta P}{\Delta V}=0$ i.e., closer to the point of MPP. Lesser step size gives less SS oscillations, but at the cost of sluggish transient response, and large step size provides faster response. However, at the cost of high SS oscillations around the MPP, which is more important during stochastic environmental conditions.

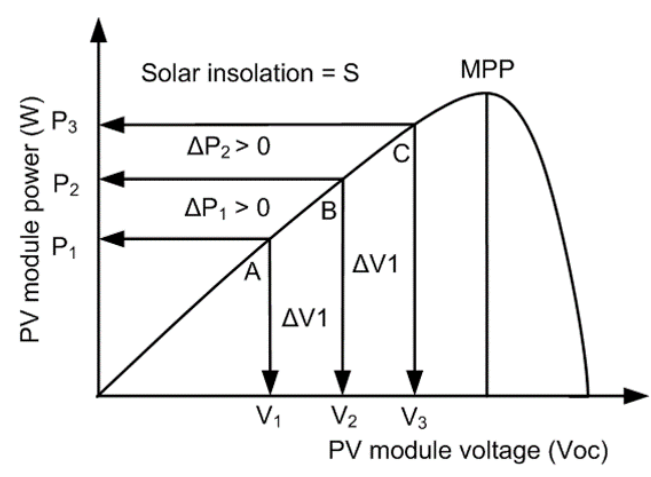

Fig. 2 P-V characteristic for P\&O MPPT

The above-mentioned shortcoming of the $\mathrm{P} \& \mathrm{O}$ scheme is compensated by the IC scheme. The algorithm for the same can be found in (Pathak and Yadav, 2019).

\section{B) IC technique}

The slope of P-V characteristic curve of PV array plays the key role of basic building block of the IC technique (Danandeh et al., 2018). The key idea behind the IC MPPT technique is a derivative of PV power w.r.t. voltage is zero at MPP. The PV module voltage is tuned relative to MPP voltage by calculation of incremental conductance $\frac{\Delta I_{p v}}{\Delta V_{p v}}$ and instantaneous conductance $\frac{I_{p v}}{V_{p v}}$ with the help of following equations (Danandeh et al., 2018):

$\left\{\begin{array}{lr}\frac{d P_{p v}}{d V_{p v}}<0 & \text { on the right of MPP } \\ \frac{d P_{p v}}{d V_{p v}}=0 & \text { at MPP } \\ \frac{d P_{p v}}{d V_{p v}}>0 & \text { on the left of MPP }\end{array}\right\}$

The power output derivative of the PV array is given as follows:

$\frac{d P_{p v}}{d V_{p v}}=\frac{d\left(I_{p v} V_{p v}\right)}{d V_{p v}}=I_{p v}+V_{p v} \frac{d I_{p v}}{d V_{p v}}=I_{p v}+V_{p v} \frac{\Delta I_{p v}}{\Delta V_{p v}}$

$\left\{\begin{array}{lr}\frac{\Delta I_{p v}}{\Delta V_{p v}}=-\frac{I_{p v}}{V_{p v}} & \text { at MPP } \\ \frac{\Delta I_{p v}}{\Delta V_{p v}}>-\frac{I_{p v}}{V_{p v}} & \text { at the left of MPP } \\ \frac{\Delta I_{p v}}{\Delta V_{p v}}<-\frac{I_{p v}}{V_{p v}} & \text { at the right of MPP }\end{array}\right\}$

The algorithm for the same can be found in (Pathak and Yadav, 2019).

\section{C) Proposed ROP\&O Technique}


The main drawbacks of the classical P\&O MPPT technique are oscillations of controller around MPP, and loss of tracking direction can be fruitfully addressed and overcome by the proposed scheme of ROP\&O MPPT. The flowchart of the ROP\&O MPPT scheme is represented in Fig. 3. The proposed scheme is rooted on classical P\&O technique with an additional advantage of having dynamically varying step size in order to reduce SS oscillations around MPP. For fruitful tracking of MPP, the larger step size is utilized when the perturbation is towards MPP, and if perturbation crosses MPP i.e., falls on the right-hand side of the P-V characteristic curve, smaller step size is utilized. The search for reference MPP voltage $\left(V_{\text {ref,mpp }}\right)$ starts with an initial step size $(\delta)$ of 0.007 . When the operating point lies on left-hand side of the P-V characteristic curve i.e., $\Delta P_{p v}>0, \delta$ is increased by multiplying it by a constant $\alpha$ (where $\alpha>1$ ), whereas in the reverse condition i.e., $\Delta P_{p v}<0, \delta$ is reduced by dividing it by $\alpha$. The main motive behind the utilization of $\alpha$ is to enhance MPP tracking speed by increasing step size besides ensuring effective tracking by reducing step size near MPP. A generic equation of reference MPP voltage of PV array is presented as:

$V_{\text {ref,mpp }}=V_{p v}(k)+\left[\operatorname{sign}\left(V_{p v}(k)-V_{p v}(k-1)\right) \times \operatorname{sign}\left(P_{p v}(k)-P_{p v}(k-1)\right)\right] \times \delta_{\text {updated }}$

where, $\mathrm{PV}$ voltage and power at $k^{\text {th }}$ iteration is $V_{p v}(k)$, and $P_{p v}(k)$ respectively, and $\delta_{\text {updated }}$ is updated value of step size. The core of proposed scheme is the use of PI controller for the BPC. Its function is to maintain the input PV voltage equal to the reference MPP voltage. The input error signal of PI controller is given as:

$e=\left(V_{r e f, m p p}-V_{p v}\right)$

The generated error signal is effectively minimized by the PI controller whose output is governed by the (11):

$u_{p i}=e\left(k_{p}+\frac{k_{i}}{s}\right)$

The $u_{p i}$ is further processed through PWM generator to generate gating pulses for IGBT of the BPC. The proposed ROP\&O MPPT scheme is benchmarked using a BPC, and the simulation parameter of the PV array is given in Table. 1. 


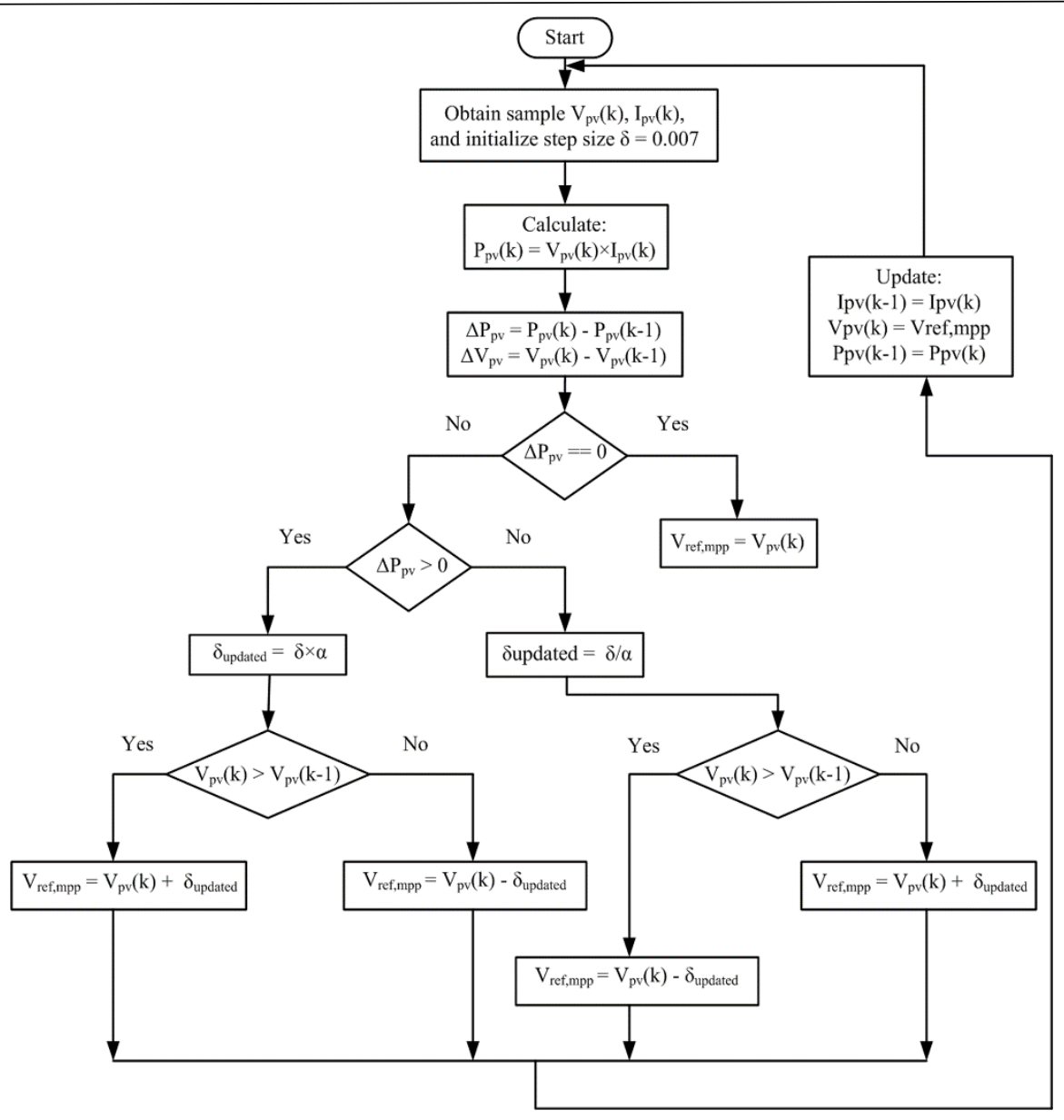

Fig. 3 Flowchart of ROP\&O MPPT

It is considered that the BPC is operating in continuous current mode. Based on the state-space averaging method, the dynamics of system are presented as (Kollimalla \& Mishra 2016(b)):

$\frac{d i_{p v}}{d t}=-\frac{(1-d)}{L} v_{o}+\frac{v_{p v}}{L}$

$\frac{d v_{o}}{d t}=-\frac{(1-d)}{C} i_{p v}-\frac{1}{R C} v_{o}$

where, input voltage, input current, duty cycle, and output voltage of the boost converter are $v_{p v}, i_{p v}, d$, and $v_{o}$, respectively.

Table 1: Numerical values of PV for simulation (Pathak and Yadav 2019)

\begin{tabular}{cl}
\hline Parameters & Values \\
\hline $\boldsymbol{P}_{\boldsymbol{m p} \boldsymbol{p}}$ & $200 \mathrm{~W}$ \\
$\boldsymbol{V}_{\boldsymbol{o c}}$ & $32.724 \mathrm{~V}$ \\
$\boldsymbol{I}_{\boldsymbol{s c}}$ & $8.21 \mathrm{~A}$ \\
$\boldsymbol{V}_{\boldsymbol{m p} \boldsymbol{p}}$ & $25.92 \mathrm{~V}$ \\
$\boldsymbol{I}_{\boldsymbol{m p} \boldsymbol{p}}$ & $7.7 \mathrm{~A}$ \\
Cells in a module & 18 \\
$\boldsymbol{N}_{\boldsymbol{s}}$ & 3 \\
No. of cells in parallel $\left(\boldsymbol{N}_{\boldsymbol{p}}\right)$ & 1 \\
$\boldsymbol{A}$ & 1.36 \\
\hline
\end{tabular}

The transfer function (TF) of the BPC is given as (Kollimalla \& Mishra 2016(b)):

$G_{i}(s)=\frac{\left(V_{o} C\right) s+2 \cdot \frac{V_{o}}{R_{L}}}{(L C) s^{2}+\frac{L}{R_{L}} s+(1-D)^{2}}$

The TF of PI controller is represented as follows: $G_{c}(s)=k_{p}+\frac{k_{i}}{s}$. The open-loop TF (OLTF) of BPC in conjunction with the PI controller is formulated as: 
$G_{o l}(s)=G_{i}(s) \cdot G_{c}(s)$

The controller parameters are obtained from GM and PM technique, the Bode plot for uncompensated $\left(G_{i}(s)\right)$ as well as compensated $\left(G_{o l}(s)\right)$ system is presented in Fig. 4. The PM of $G_{i}(s)$ is $86.9^{\circ}$ at $22.20 \mathrm{krad} / \mathrm{sec}$ at the same time, MPPT scheme is executed at every 50 cycles of $f_{s}$. It is required to obtain a suitable PM at the gain crossover frequency of $1.25 \mathrm{krad} / \mathrm{sec}$. A PM of $75.5^{\circ}$ at $1.25 \mathrm{krad} / \mathrm{sec}$ is obtained employing PI controller i.e., $G_{o l}(s)$, the gains of the controller are calculated and valued as $k_{p}=0.002$ and $k_{i}=45.50$.

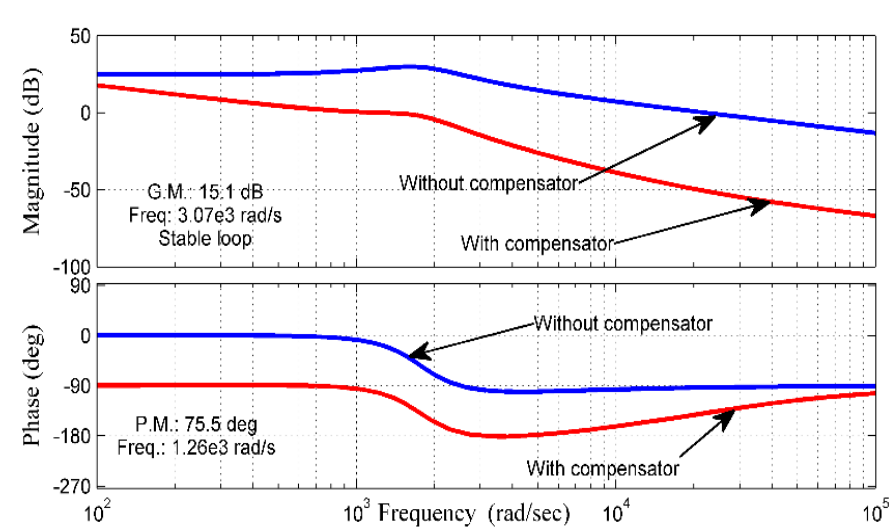

Fig. 4 Calculation of GM \& PM using bode plot

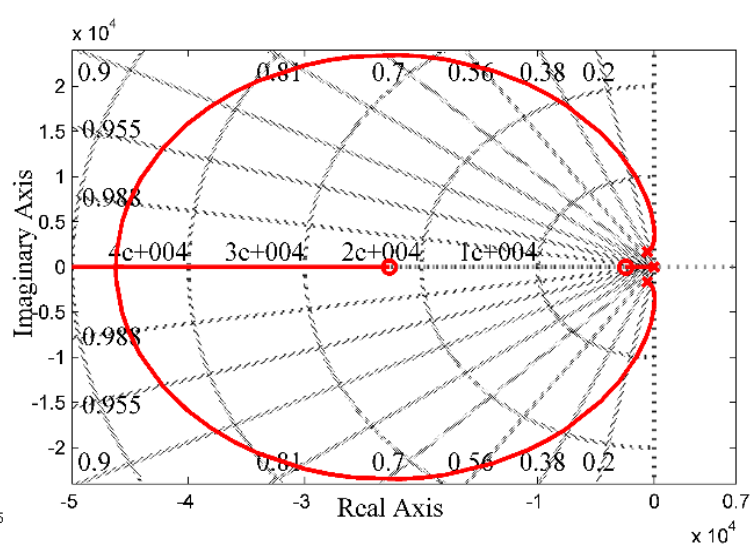

Fig. 5 Root locus of converter employing ROP\&O MPPT

To check the stability of the BPC employing ROP\&O MPPT scheme (the compensated TF $G_{o l}(s)$ consist the values obtained via the implementation of proposed MPPT), analysis is done using root locus technique, and the root locus diagram of $G_{o l}(s)$ is shown in Fig. 5. After analyzing the root locus diagram, a conclusion is drawn that the closed-loop poles for designed PM occur at $p_{1}=-636$ and $p_{2,3}=-319 \pm j 1940$, ensuring the system stability. The error rates of the proposed MPPT technique along with classical P\&O and classical IC can be calculated using the following mathematical formulation (Rezk et al., 2019):

MAPE $=\frac{1}{N} \sum\left(\frac{\mid \text { Actual }- \text { Forecasted } \mid}{\mid \text { Actual } \mid}\right) \times 100 \%$, MRE $=\frac{\mid \text { Actual }- \text { Estimeted } \mid}{\mid \text { Actual } \mid}$, and RMSE $=\sqrt{\frac{\sum_{i=1}^{n}(\text { Predicted }- \text { Achieved })^{2}}{N}}$ where, $N$ is the number of samples.

\section{Results and Discussion}

A complete model of the PV system, in conjunction with the proposed ROP\&O MPPT scheme, is presented in Fig. 1. It consists of a PV panel, BPC, connected load is resistive in nature, and an MPPT controller. The sampling time $\left(T_{p}\right)$ for the MPPT scheme is obtained as (Femia et al., 2005):

$T_{p} \geq T_{a} \cong-\frac{1}{\xi \omega_{n}} \times \ln (\in)$

where, $\omega_{n}=\frac{1}{\sqrt{L C_{i n}}}, \xi=\frac{1}{2 R_{L}} \times \sqrt{\frac{L}{C_{i n}}}, C_{i n}(=1 m F)$ is the input capacitor of boost converter, and $\in=0.10$. In this work, $T_{p}$ is set as $0.0299 \mathrm{sec}$. The pattern of insolation change in all the considered five states is shown in Fig. 7(a), varying in between $400-1000 \mathrm{~W} / \mathrm{m}^{2}$ at $25^{\circ} \mathrm{C}$ temperature. Fig. 7(b) shows the simulation comparison of maximum obtainable power from $\mathrm{PV}$ employing $\mathrm{ROP} \& \mathrm{O}$, conventional $\mathrm{P} \& \mathrm{O}$, and conventional IC as MPPT scheme. 


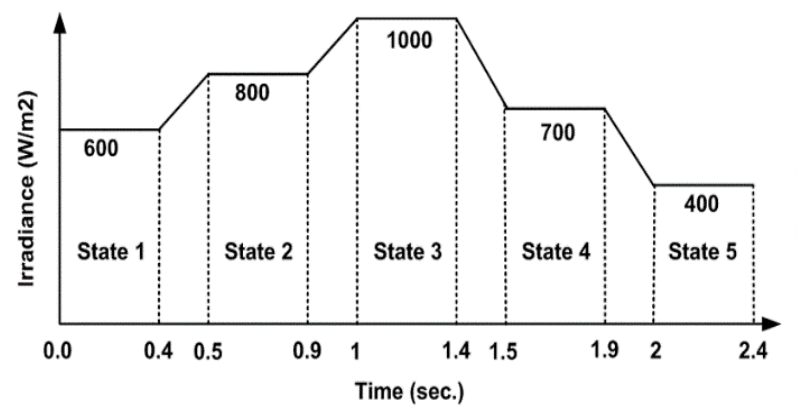

(a)

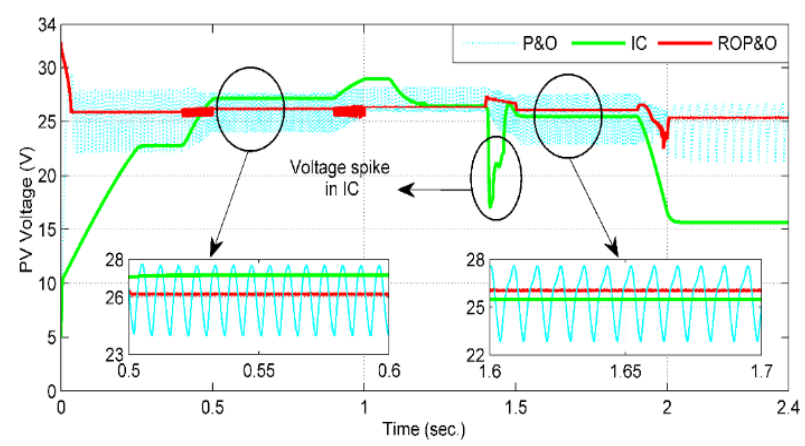

(c)

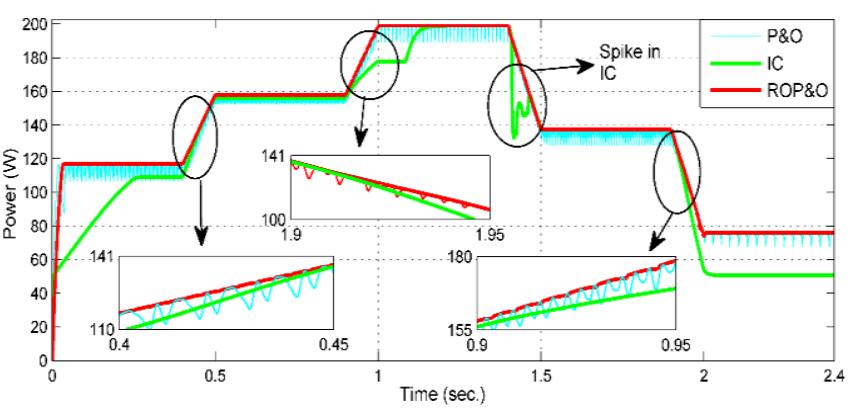

(b)

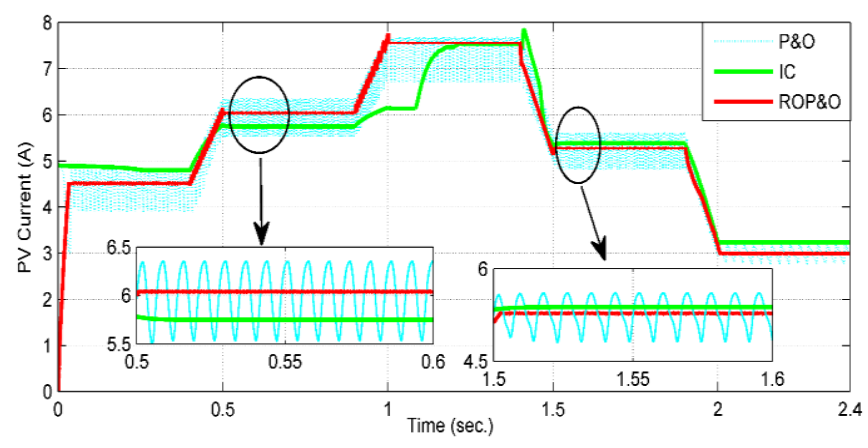

(d)

Fig. 7 (a) Insolation profile, PV (b) Power (c) Voltage (d) Current employing P\&O, IC and ROP\&O MPPT technique

From Fig. 7(b) it can be concluded that the IC technique takes the longest time of 270 ms to track MPP, P\&O technique takes $80 \mathrm{~ms}$, whereas the ROP\&O technique takes only $18 \mathrm{~ms}$. Ripple content around the MPP is largest in the case of $\mathrm{P} \& \mathrm{O}$ technique, which is considerably reduced in the case of IC technique but at the cost of being unable to achieve the MPP, and almost zero ripple content for ROP\&O technique with easy tracking of MPP under states 1 to 5 of insolation level. In each state, the power trajectory is clearly represented in Fig. 8(a)-(e). In the transition from state 3 to state 4 at time $t=1.1 \mathrm{~s}$ to $t=1.4 \mathrm{~s}$ there is a sudden ripple i.e., loss of tracking direction in case of IC technique as depicted in Fig. 7(b). Moreover, at a low insolation level of $400 \mathrm{~W} / \mathrm{m}^{2}$ i.e., in the state $5 \mathrm{IC}$ technique, it fails to achieve the MPP with the $\eta$ falling as low as $66.23 \%$. The $\eta$ of $\mathrm{P} \& \mathrm{O}$ technique is in between $93.37 \%$ to $97.81 \%$, for IC technique it is in between $66.23 \%$ to $98.55 \%$, whereas for proposed ROP\&O it is between $99.06 \%$ to $99.80 \%$.

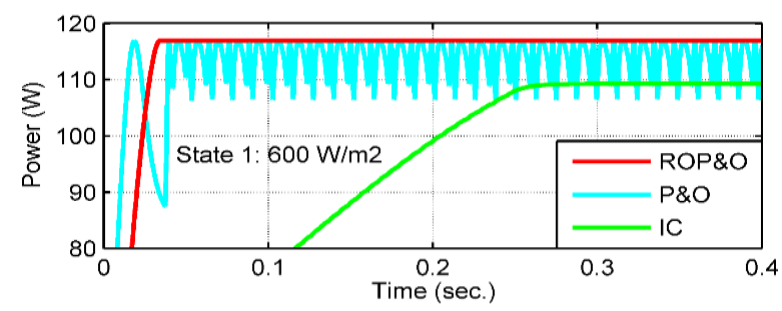

(a)

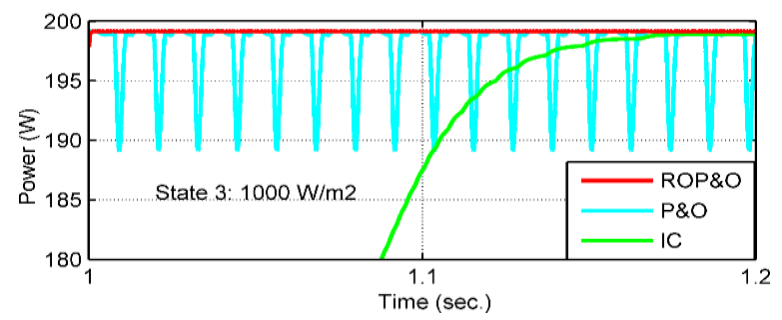

(c)

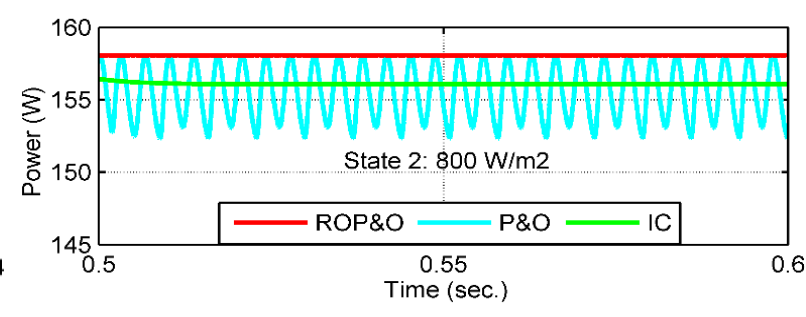

(b)

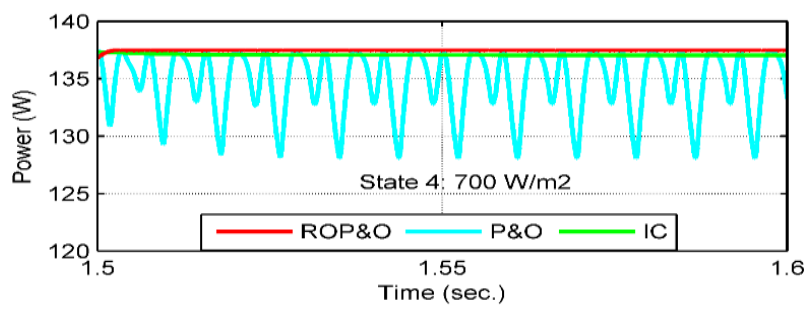

(d) 


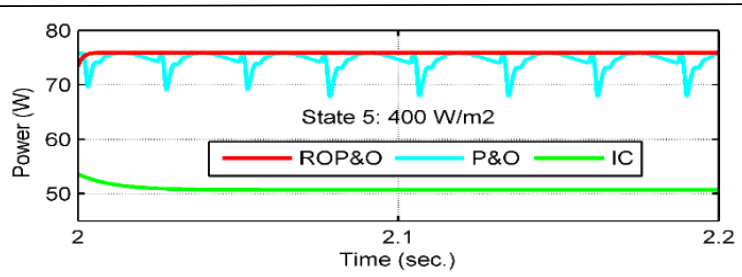

(e)

Fig. 8 (a)-(e) PV power in all considered states

Fig. 9(a) shows the graphical representation of $\eta$ for all three MPPT techniques. Comparative analysis of the simulation results of the $V_{p v}$ and $I_{p v}$ is clearly depicted in Fig. 7(c) and (d), which conclude that the ripple content is very large in the case of the P\&O technique, decreased in case of IC technique, and almost zero in case of the proposed ROP\&O technique. While analyzing the voltage ripple the values are $6 \mathrm{~V}, 3.5 \mathrm{~V}, 2.5 \mathrm{~V}, 4.5 \mathrm{~V}$ and $5 \mathrm{~V}$ in states 1 to 5 respectively for $\mathrm{P} \& \mathrm{O}$ scheme. In case of IC scheme voltage ripples are $0.4 \mathrm{~V}, 0.2 \mathrm{~V}, 2.2 \mathrm{~V}, 0.2 \mathrm{~V}$ and $0.2 \mathrm{~V}$ in states 1 to 5 respectively. While considering the proposed technique voltage ripples are almost negligible as depicted in Table 2. So, the maximum voltage ripple content in the case of $\mathrm{P} \& \mathrm{O}$ technique is $6 \mathrm{~V}$, which is $2.2 \mathrm{~V}$ and $0.08 \mathrm{~V}$ in the case of IC and ROP\&O techniques, respectively. The current ripple contents are $0.9 \mathrm{~A}, 0.8 \mathrm{~A}, 0.7 \mathrm{~A}, 0.8 \mathrm{~A}$ and $0.8 \mathrm{~A}$ in states 1 to 5 respectively for the $\mathrm{P} \& \mathrm{O}$ scheme. In case of IC technique, the ripple contents in current are $0.12 \mathrm{~A}, 0.1 \mathrm{~A}, 0.4 \mathrm{~A}, 0.11 \mathrm{~A}$ and $0.11 \mathrm{~A}$ in states 1 to 5 respectively as presented in Table 2. For the ROP\&O MPPT scheme the ripple contents in current are negligible for all the considered states. So, it can be noted that the maximum current ripple is $0.9 \mathrm{~A}, 0.4 \mathrm{~A}$, and negligible in the case of P\&O, IC, and ROP\&O MPPT techniques, respectively. The maximum value of MAPE, MRE, and RMSE is $6.877 \%$, $0.117,8.807$ respectively in case of $\mathrm{P} \& \mathrm{O}$ technique, $31.815 \%, 0.3376,24.545$ respectively in case of IC technique and $1.35 \%, 0.0146,1.5$ respectively in case of proposed ROP\&O technique. From the table 2 it can be concluded that the error rates are minimum in case of ROP\&O MPPT scheme.

Fig. 9(b) illustrates the comparative graphical representation of tracking power loss for all the three MPPT techniques in all the five considered states. Their power loss $\left(P_{l o s s}\right)$ is defined by:

$P_{\text {loss }}=\frac{\sum P_{\max }(t)-\sum P_{p v}(t)}{\sum P_{\max }(t)}$

where, $P_{\text {max }}=\left(P_{m p p}\right)$ is the maximum power under given irradiation, $P_{p v}$ is the actual power extracted, and $t$ refers to the tracking time required to reach the MPP by different MPPT schemes. Fig. 9(b) clearly indicates that the calculated power loss is least in the case of the proposed ROP\&O MPPT scheme in comparison to conventional P\&O and IC schemes. Thus, it can be concluded that the proposed technique results in almost complete elimination of oscillations, and the power loss through this technique is least along with the highest tracking efficacy under each considered state.

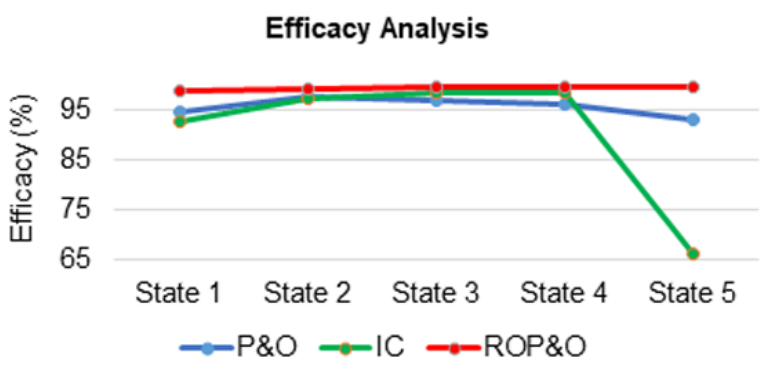

(a)

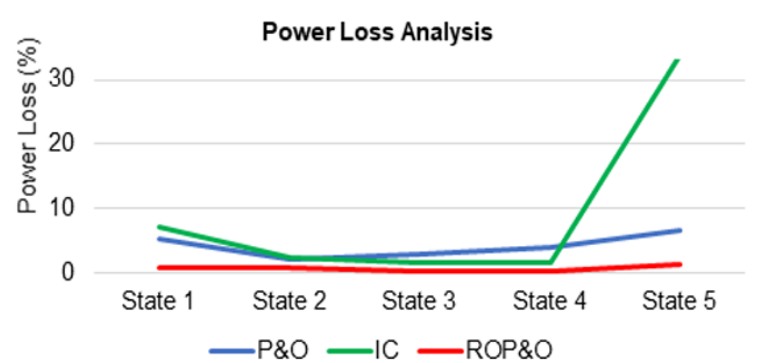

(b)

Fig. 9 Comparative analysis (a) Tracking efficacy (b) Power loss

Table 2: Vivid analysis of P\&O, IC, and proposed ROP\&O MPPTs 


\begin{tabular}{llllll}
\hline Average Actual Power (W) & & & & \\
P\&O & 111.7 & 156.5 & 194 & 132.7 & 71.9 \\
IC & 109.5 & 156.2 & 197 & 136 & 51 \\
ROP\&O & 116.9 & 158.8 & 199.6 & 137.5 & 76 \\
Tracking Efficacy (\%) & & & & \\
P\&O & 94.67 & 97.81 & 97.00 & 96.16 & 93.37 \\
IC & 92.80 & 97.62 & 98.50 & 98.55 & 66.23 \\
ROP\&O & 99.06 & 99.25 & 99.80 & 99.65 & 99.70 \\
PV Voltage Ripple (V) & & & & \\
P\&O & 6 & 3.5 & 2.5 & 4.5 & 5 \\
IC & 0.4 & 0.2 & 2.2 & 0.2 & 0.2 \\
ROP\&O & 0.08 & 0.07 & 0.06 & 0.08 & 0.05 \\
PV Current Ripple (A) & & & & \\
P\&O & 0.9 & 0.8 & 0.7 & 0.8 & 0.8 \\
IC & 0.12 & 0.1 & 0.4 & 0.11 & 0.11 \\
ROP\&O & Negligible & Negligible & 0.02 & 0.02 & Negligible \\
Convergence Time (ms) & & & & \\
P\&O & 80 & 70 & 70 & 80 & 80 \\
IC & 270 & 40 & 150 & 50 & 60 \\
ROP\&O & 18 & 10 & 1 & 4 & 3 \\
Error rate at MPP & & & & \\
(MAPE, MRE, RMSE) & & & & \\
P\&O (MAPE) & $6.67 \%$ & $3.54 \%$ & $2.34 \%$ & $3.985 \%$ & $6.887 \%$ \\
P\&O (MRE) & 0.1017 & 0.05 & 0.055 & 0.07246 & 0.117 \\
P\&O (RMSE) & 8.807 & 6.245 & 6.48 & 6.181 & 6.233 \\
IC (MAPE) & $7.627 \%$ & $2.125 \%$ & $1.525 \%$ & $0.85 \%$ & $31.815 \%$ \\
IC (MRE) & 0.0762 & 0.02375 & 0.02 & 0.00954 & 0.3376 \\
IC (RMSE) & 8.753 & 3.423 & 3.67 & 1.184 & 24.545 \\
ROP\&O (MAPE) & $0.94 \%$ & $0.592 \%$ & $0.23 \%$ & $0.653 \%$ & $1.35 \%$ \\
ROP\&O (MRE) & 0.00953 & 0.0059 & 0.00233 & 0.0066 & 0.0146 \\
ROP\&O (RMSE) & 1.08 & 1.5 & 0.862 & 0.904 & 0.585 \\
\hline & & & & \\
\hline
\end{tabular}

Table 3: Comparative analysis of proposed method with other methods

\begin{tabular}{|c|c|c|c|c|c|c|c|}
\hline MPPT scheme & Complexity & Efficacy & $\begin{array}{l}\text { Oscillation } \\
\text { around MPP }\end{array}$ & $\begin{array}{l}\text { Convergence } \\
\text { speed }\end{array}$ & Cost & $\begin{array}{l}\text { Periodic } \\
\text { tuning }\end{array}$ & $\begin{array}{l}\text { Input } \\
\text { variables }\end{array}$ \\
\hline $\begin{array}{l}\text { Open circuit voltage } \\
\text { (Reisi et al., 2013) }\end{array}$ & Less & Less & $\mathrm{Y}$ & Moderate & Cheap & $\mathrm{N}$ & Voltage \\
\hline $\begin{array}{l}\text { Short circuit current } \\
\text { (Reisi et al., 2013) }\end{array}$ & Medium & Less & $\mathrm{Y}$ & Moderate & Cheap & $\mathrm{N}$ & Current \\
\hline $\begin{array}{l}\text { Artificial neural } \\
\text { network (Pathak et al., } \\
\text { 2020) }\end{array}$ & Larger & Larger & $\mathrm{Y}$ & Fast & Expensive & $\mathrm{Y}$ & $\begin{array}{l}\text { User } \\
\text { knowledge }\end{array}$ \\
\hline $\begin{array}{l}\text { P\&O (fixed step) } \\
\text { (Reisi et al., 2013) }\end{array}$ & Less & Less & $\mathrm{Y}$ & Less & Moderate & $\mathrm{N}$ & $\begin{array}{l}\text { Current and } \\
\text { Voltage }\end{array}$ \\
\hline $\begin{array}{l}\text { P\&O (variable step) } \\
\text { (Reisi et al., 2013) }\end{array}$ & Medium & Larger & $\mathrm{Y}$ & Fast & Moderate & $\mathrm{N}$ & $\begin{array}{l}\text { Current and } \\
\text { Voltage }\end{array}$ \\
\hline $\begin{array}{l}\text { FL (Pathak et al., } \\
\text { 2020) }\end{array}$ & Larger & Larger & $\mathrm{Y}$ & Fast & Expensive & $\mathrm{Y}$ & $\begin{array}{l}\text { User } \\
\text { knowledge }\end{array}$ \\
\hline $\begin{array}{l}\text { FL-DPID (Pathak and } \\
\text { Yadav, 2019) }\end{array}$ & $\begin{array}{l}\text { Larger but } \\
\text { lower than } \\
\text { conventional } \\
\text { FL based }\end{array}$ & $\begin{array}{l}\text { Larger but low at } \\
\text { low irradiance } \\
\text { level }\end{array}$ & $\mathrm{N}$ & Fast & Expensive & $\mathrm{Y}$ & $\begin{array}{l}\text { User } \\
\text { knowledge }\end{array}$ \\
\hline $\begin{array}{l}\text { IC (Safari \& Mekhilef, } \\
\text { 2010) }\end{array}$ & Medium & Larger & $\mathrm{Y}$ & Fast & Moderate & $\mathrm{N}$ & $\begin{array}{l}\text { Current and } \\
\text { Voltage }\end{array}$ \\
\hline $\begin{array}{l}\text { Back stepping control } \\
\text { (Pathak et al., 2020) }\end{array}$ & Medium & Larger & $\mathrm{Y}$ & Fast & Moderate & $\mathrm{N}$ & $\begin{array}{l}\text { Temperature } \\
\text { and Irradiance }\end{array}$ \\
\hline $\begin{array}{l}\text { Improved FL based (Rezk } \\
\text { et al., 2019) }\end{array}$ & Medium & Larger & $\mathrm{N}$ & Fast & Expensive & $\mathrm{Y}$ & $\begin{array}{l}\text { Voltage, } \\
\text { Temperature } \\
\text { and } \\
\text { Irradiance }\end{array}$ \\
\hline Proposed ROP\&O & Less & Larger & $\mathrm{N}$ & Fast & Moderate & $\mathrm{N}$ & $\begin{array}{l}\text { Current and } \\
\text { Voltage }\end{array}$ \\
\hline
\end{tabular}

\section{Validation of proposed MPPT technique via grid connection}

Three phase grid is considered to benchmark the ROP\&O MPPT technique, and the operation is chosen at unity power factor. A three-phase voltage source inverter (VSI) is utilized to convert a 500V DC voltage in $260 \mathrm{~V}$ AC. In order to affiliate the grid and VSI a transformer of rating $100 \mathrm{kVA}, 260 \mathrm{~V} / 20 \mathrm{kV}$ is used. The three-phase grid comprises of $120 \mathrm{kV}$ transmission systems with $20 \mathrm{kV}$ distribution feeders. The synchronization of VSI is performed via conventional voltageoriented control (VOC) technique (Pathak et al., 2018). The performance evaluation of the ROP\&O MPPT scheme has been investigated in terms of THD under considered irradiance levels. The schematic arrangement of grid integration 
with double stage conversion is shown in Fig. 10. Fig. 11 (a) and (b), show the grid voltage and current respectively which reveals the proper synchronization and effectiveness of ROP\&O MPPT scheme. The THD analysis of the grid current is analyzed and depicted in Fig. 12 (a) and (b) under transient as well as the steady state condition. In the SS condition $\left(1000 \mathrm{~W} / \mathrm{m}^{2}\right)$ in the obtained THD is $2.84 \%$ while in case of transient condition $\left(800-1000 \mathrm{~W} / \mathrm{m}^{2}\right)$ it is $3.11 \%$. The range of $5 \%$ given by the IEEE 519 standard is fruitfully satisfied by the THD obtained in case of proposed scheme as revealed in Fig. 12.

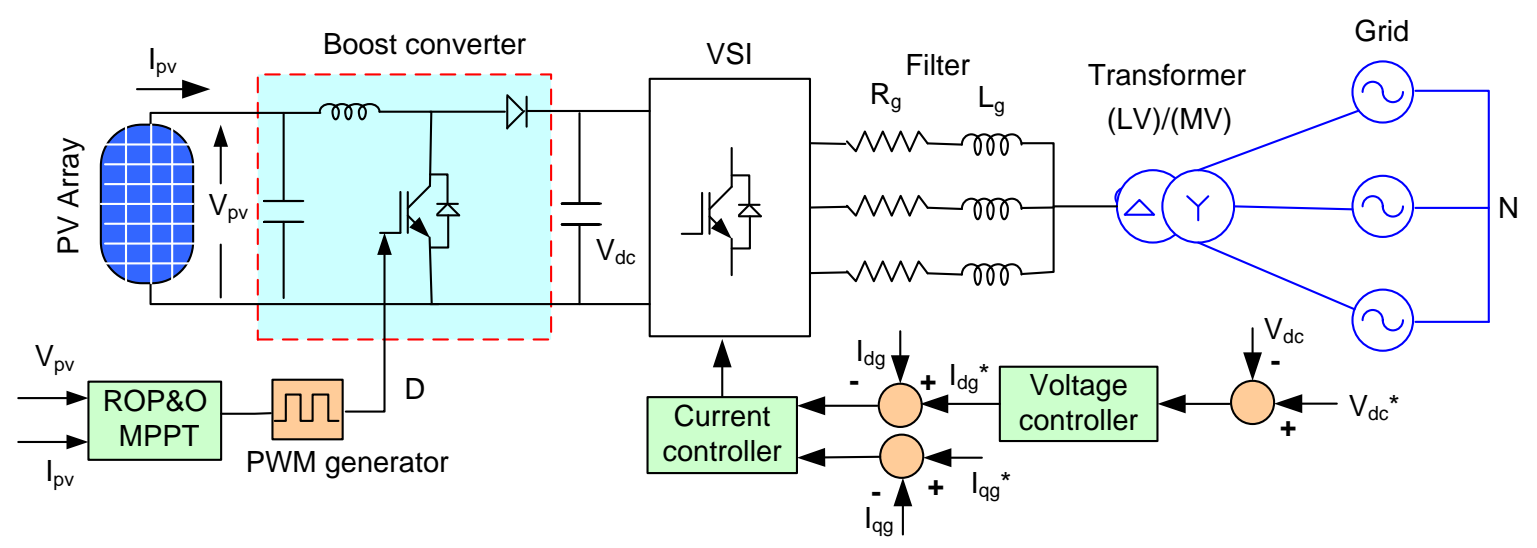

Fig. 10 Schematic diagram of grid integrated PV

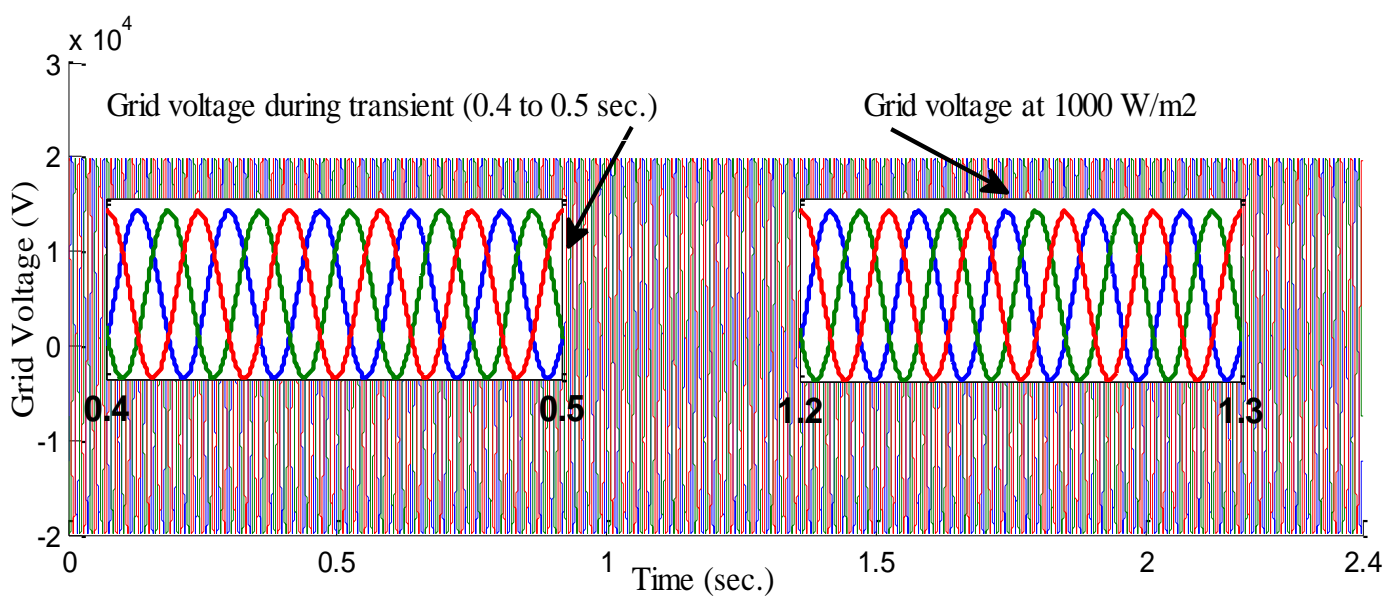

(a)

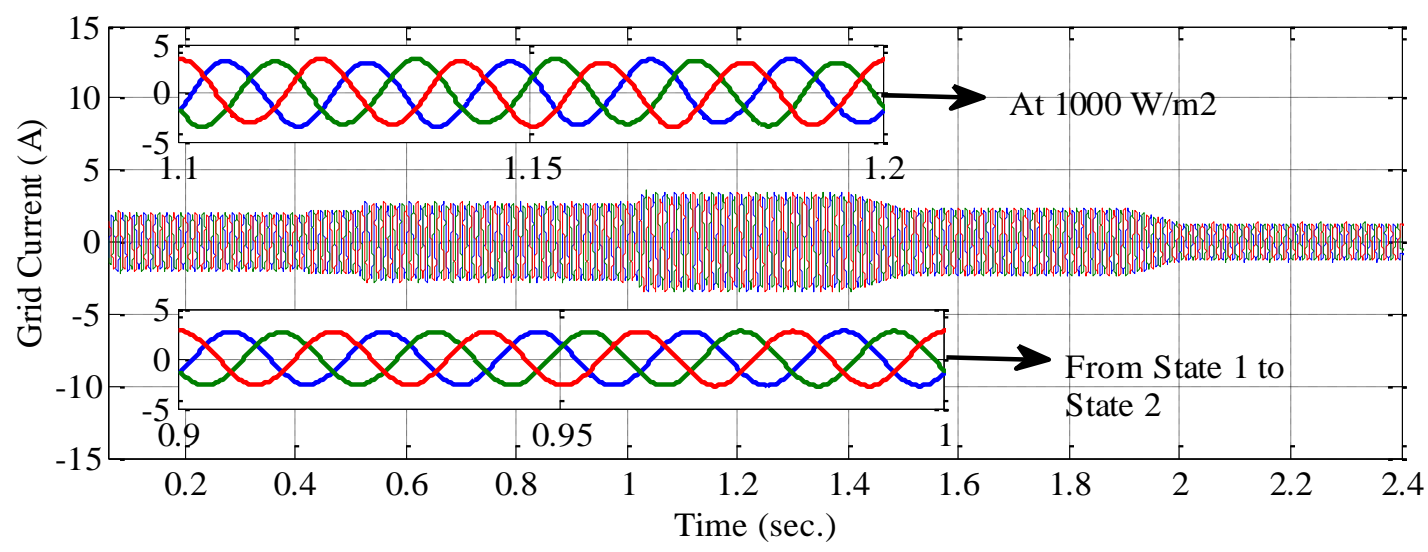

(b)

Fig. 11 Grid (a) voltage and (b) current 


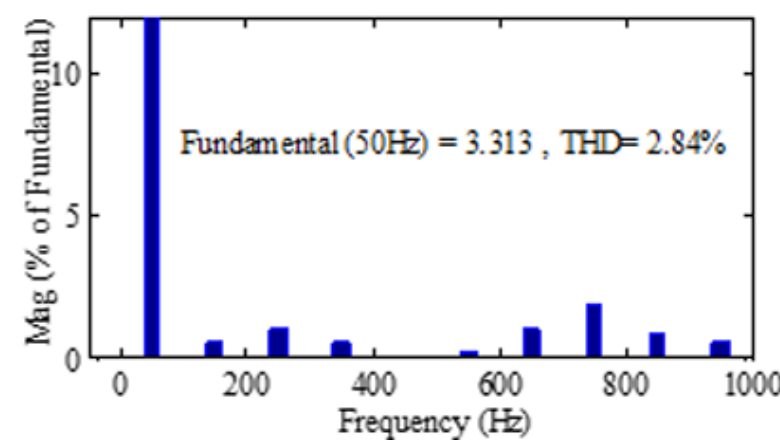

(a)

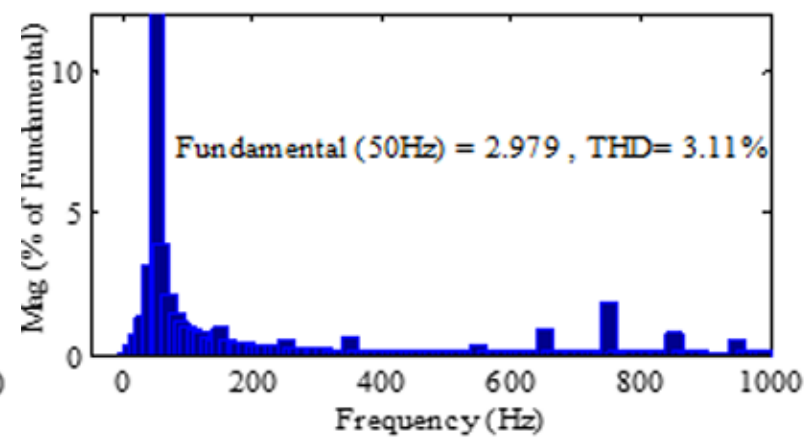

(b)

Fig. 12 THD of grid current (a) At $1000 \mathrm{~W} / \mathrm{m}^{2}$, (b) Transient region $800-1000 \mathrm{~W} / \mathrm{m}^{2}$

\section{Conclusion}

This work proposes an ROP\&P MPPT scheme, and a vivid comparative analysis of the performance parameters with commonly used schemes such as classical P\&O and classical IC is obtained. Under the stochastic nature of atmospheric conditions, the proposed MPPT scheme provides better results with respect to the other considered schemes to determine MPP. The ROP\&O MPPT scheme's tracking efficacy lies in between $99.06 \%$ to $99.80 \%$, while P\&O is in between $93.37 \%$ to $97.81 \%$, and IC is in between $66.23 \%$ to $98.55 \%$ for all the considered states. The oscillations around MPP are negligible, and tracking power loss is lowest in the proposed MPPT technique in comparison to the P\&O and IC scheme. This brings the scheme to the leading in nature in terms of efficacy. The time taken by the proposed MPPT scheme to reach SS is $0.018 \mathrm{sec}$. which is about five times faster than the conventional P\&O technique and fifteen times faster than the conventional IC technique. The error rates at MPP are lowest in the ROP\&O MPPT scheme compared to P\&O and IC techniques. Also, the proposed technique is validated using three phase grid and the obtained results show the transmitted grid current power quality is satisfying the IEEE 519 standard. And the stablished operation of ROP\&O scheme is revealed by the time domain analysis and the technique is easy to implement with reduced computational load on the system. The major drawback of proposed technique is that it is not suitable for partial shading conditions. The future scope of this work is to design ROP\&O based MPPT for partial shading conditions.

\section{References}

Abdelsalam, A. K, Massoud A. M, Ahmed, S \& Enjeti P. 2011. High performance adaptive perturb and observe MPPT technique for photovoltaic-based microgrids. IEEE Transactions on Power Electronics, 26(4), 1010-21.

Ahmed, J. \& Salam, Z. 2016. A modified P\&O maximum power point tracking method with reduced steady-state oscillation and improved tracking efficiency. IEEE Transactions on Sustainable Energy, 7(4):1506-14.

Ali, A. I. M., Sayed, M. A. \& Mohamed, E. E. M. 2018. Modified efficient perturb and observe maximum power point tracking technique for grid-tied PV system. International Journal of Electric Power and Energy System 99:192-202.

Alik, R. \& Jusoh, A. 2017. Modified perturb and observe (P\&O) with checking algorithm under various solar irradiation. Solar Energy, 148:128-39.

Danandeh, M. A. \& Mousavi, S. M. 2018. A new architecture of INC-fuzzy hybrid method for tracking maximum power point in PV cells. Solar Energy, 171:692-703.

Ding K, Bian X, Liu H \& Peng T. 2012. A MATLAB-simulink based PV module model and its application under conditions of nonuniform irradiance. IEEE Transactions on Energy Conversion, 27(4), 864-72.

Elgendy, M. A., Zahawi, B. \& Atkinson, D. J. 2013. Assessment of the incremental conductance maximum power point tracking algorithm. IEEE Transactions on Sustainable Energy, 4(1):108-17. 
Elgendy, M. A., Zahawi, B. \& Atkinson, D. J. 2012. Assessment of perturb and observe MPPT algorithm implementation techniques for PV pumping applications. IEEE Transactions on Sustainable Energy, 3(1), 21-33.

Femia, N., Petrone, G., Spagnuolo, G. \& Vitelli M. 2005. Optimization of perturb and observe maximum power point tracking method. IEEE Transactions on Power Electronics, 20(4):963-73.

Killi, M. \& Samanta, S. 2015. Modified perturb and observe MPPT algorithm for drift avoidance in photovoltaic systems. IEEE Transactions on Industrial Electronics, 62(9), 5549-59

Kollimalla, S. K. \& Mishra, M. K. 2014(a). Variable perturbation size adaptive P\&O MPPT algorithm for sudden changes in irradiance. IEEE Transactions on Sustainable Energy, 5(3), 718-28.

Kollimalla, S. K. \& Mishra, M. K. 2014(b). A novel adaptive P\&O MPPT algorithm considering sudden changes in the irradiance. IEEE Transactions on Energy Conversion, 29(3), 602-10.

Kumar, N., Hussain, I., Singh, B. \& Panigrahi, B., K. 2018. Framework of maximum power extraction from solar PV panel using self-predictive perturb and observe algorithm. IEEE Transactions on Sustainable Energy, 9(2):895-903.

Li, X., Wen, H., Jiang, L., Xiao, W., Du, Y. \& Zhao, C. 2016. An improved MPPT method for PV system with fastconverging speed and zero oscillation. IEEE Transactions on Industry Applications, 52(6), 5051-5064.

Pandey A, Dasgupta N \& Mukerjee A.K. 2008. High performance algorithms for drift avoidance and fast tracking in solar MPPT system. IEEE Transactions on Energy Conversion, 23(2), 681-89.

Pathak, P. K., Yadav, A. K. \& Tyagi, P. 2018. Design of three phase grid tied solar photovoltaic system based on three phase VSI. In 2018 8th IEEE India International Conference on Power Electronics (IICPE),1-6).

Pathak, P. K., Yadav, A. K. \& Alvi, P. A. 2020. Advanced solar MPPT techniques under uniform and non-uniform irradiance: a comprehensive review. Journal of Solar Energy Engineering, 142(4): 040801-1-26.

Pathak, P. K., Yadav, A. K. \& Alvi, P. A. 2021(a). A state-of-the-art review on shading mitigation techniques in solar photovoltaics via meta-heuristic approach. Neural Computing and Applications, 1-39.

Pathak, P.K. \& Yadav, A.K. 2019. Design of battery charging circuit through intelligent MPPT using SPV system. Sol. Energy, 178: 79-89.

Pathak, P. K., Padmanaban, S., Yadav, A. K., Alvi, P. A. \& Khan, B. 2021(b). Modified incremental conductance MPPT algorithm for SPV-based grid-tied and stand-alone systems. IET Generation, Transmission \& Distribution, 1-16.

Paz, F. \& Ordonez, M. 2014. Zero oscillation and irradiance slope tracking for photovoltaic MPPT. IEEE Transactions on Industrial Electronics, 61(11), 6138-47.

Ramesh, M., Yadav, A. K. \& Pathak, P. K. 2021 (a). An extensive review on load frequency control of solar-wind based hybrid renewable energy systems. Energy Sources, Part A: Recovery, Utilization, and Environmental Effects, 1-25.

Ramesh, M., Yadav, A. K. \& Pathak, P. K. 2021(b). Intelligent adaptive LFC via power flow management of integrated standalone micro-grid system. ISA Transactions, 112:234-250.

Reisi, A. R., Moradi, M. H. \& Jamasb, S. 2013. Classification and comparison of maximum power point tracking technique for photovoltaic system: a review. Renewable Sustainable Energy Reviews, 19:433-43.

Rezk, H., Aly, M., Al-Dhaifallah, M. \& Shoyama, M. 2019. Design and hardware implementation of new adaptive fuzzy logic-based MPPT control method for photovoltaic applications. IEEE Access, 7:106427-106438.

Safari, S. \& Mekhilef, S. 2010. Simulation and hardware implementation of incremental conductance MPPT with direct control method using Cuk converter. IEEE Transactions on Industrial Electronics, 58(4):1154-61.

Soon, T. K. \& Mekhilef, S. 2015. A fast-converging MPPT technique for photovoltaic system under fast-solar irradiation and load resistance. IEEE Transactions on Industrial Informatics, 11(1):176-86.

Zhang, F., Thanapalan, K., Procter, A., Carr, S. \& Maddy, J. 2013. Adaptive hybrid maximum power point tracking method for a photovoltaic system. IEEE Transactions on Energy Conversion, 28(2), 353-360. 